Satoshi Tsurusaki ${ }^{1}$, Satoru Kidoaki ${ }^{2}$, ('Department of Applied Chemistry, Kyushu University ${ }^{2}$ Division of Biomolecular Chemistry, Institute for Materials Science and Engineering, Kyushu University)

Adherent cells sense the micro-mechanical gradient of substrate stiffness, and show the directional movements toward harder region called mechanotaxis Based on the originally-developed photolithographic elasticity micropatterning technology, we have revealed the essential condition to induce mechanotaxis to be in designing the sharp elasticity boundary with enough high elasticity jump and narrower boundary width than adhesion area for a single cell. Several driving forces of the mechanotaxis have been discussed in the literatures so far; such as unbalanced distribution of traction forces, difference in turn over rate and stability of each focal adhesion between hard and soft regions. To elucidate the mechanism of mechanotaxis, it is essential to analyze the temporal behaviors of the traction forces and focal adhesion structure in detail. In this study, to address this issue, traction force dynamics in the process of mechanotaxis was investigated on the gels with the sharp elasticity boundary. Fluorescent micro beads were embedded in a photocurable elasticity-tunable styrenated gelatinous gel surface, temporal change of beads displacement derived from cell movement was observed, and traction force dynamics was visualized by analyzing time-course of the beads displacement. It was found that traction forces in high elasticity field were significantly larger than those in low elasticity field. The features in the traction force dynamics observed around the elasticity boundary are discussed.

\section{225 非対称弾性勾配ゲルを用いた細胞運動の長距離整流化}

Long distance rectification of cell movement on the asymmetric elastic-gradient gels

Hiroyuki Sakashita ${ }^{1}$, Satoru Kidoaki ${ }^{2},\left({ }^{1}\right.$ Grauate School of Engineering, Kyushu university: ${ }^{2}$ Institute for Materials Chemistry and Engineering, Kyushu university)

Cell senses the micro-mechanical conditions of their surrounding matrix, and alters direction of their movement. For example, the directional cell movement toward a harder region of substrate, so-called mechanotaxis, is the cellular response to surface elasticity gradient. In our previous study, by utilizing the photolithographic elasticity miropatterning for cell adhesive hydrogels, the essential conditions to induce cell mechanotaxis was clarified to be the sharp elasticity jump within the area where single cell adheres. Given these findings, mechanotaxis is a kind of local cell behavior around elasticity boundary, and can be said to be inadequate in principle for inducing long distance directional cell movement as seen in other taxis. However, is it really impossible to induce mechanotaxis over the long distance? In this study, we demonstrate long-ranged mechanotaxis on the well-designed microelastic-patterned gels. We have constructed the saw-like asymmetric elastic gradient gels with coupled patterns of sharp and diffuse elasticity boundaries using photocurable styrenated gelatin, which can be expected to rectify random cell movement into a certain direction at each unit of the patterns. Optimizing the saw-like asymmetric elastic gradient patterns (degree of elastic jump, pitch or shape of the patterns, introduction of perpendicular soft lane, etc), the long distance rectification of cell movement was successfully induced. The principled design concept for the asymmetric elastic gradient patterns is discussed.

\section{$1 P 226$ 新型インフルエンザウイルスの感染行動 Infectious behavior of novel influenza $A$ virus \\ Tatsuya Sakai, (Department of Microbiology, Kawasaki Medical School)}

Influenza virus moves on host cell surfaces to infect to the cell efficiently. Influenza virus invades a host cell through cellular endocytic processes. Because endocytosis occurs in limited areas of cell surface, the virus needs to move to the endocytic areas. In my previous study, I found that influenza virus moves on the cell surface by using virus membrane proteins: hemagglutinin and neuraminidase. Hemagglutinin binds to sialoglycan on cell surface and neuraminidase is a hydrolytic enzyme destroying sialoglycan. By exchanging bindings between hemagglutinin and sialoglycan, virus moves on cell surfaces, and neuraminidase facilitates virus moving by preventing hemagglutinin from binding to same sialoglycan molecules repeatedly. The virus moves by two different manners: rolling and sliding. While rolling, virus maintains some bindings between virus hemagglutinin and cell sialoglycan. In contrast, while sliding, virus does not interact to the cell surface. Patterns of virus motion (behavior patterns) are dependent on virus strains. Human influenza viruses move frequently by sliding, whereas avian viruses move dominantly by rolling. I think that virus behavior is one of determinants of virus host specificity. Here, I report about behavior of novel influenza A virus (H1N1). The novel virus has originated in swines and caused worldwide pandemic 2009-2010. The behavior pattern of the novel virus, unexpectedly, resembles that of avian virus rather than human virus.
Two dimensional motility of individual diatom cells on a glass surface.

Ayumu Murase ${ }^{1}$, Yosuke Kubota ${ }^{1}$, Shigeki Mayama ${ }^{2}$, Kazuo Umemura ${ }^{1},\left({ }^{1}\right.$ Faculty of Science, Tokyo University of Science: ${ }^{2}$ Faculty of Education, Tokyo Gakugei University)

Diatoms are one of the major photosynthetic planktons found in seas, rivers, and lakes. Diatoms produce $25 \%$ of the oxygen on the earth. Furthermore, fossilized diatoms, i.e., diatomites, have been widely used in various industrial applications such as filters, building materials, and carriers for chromatography because of their unique nanoporous structures. Although the diatom is one of the well-known planktons, the motility of diatoms has not yet been well understood. Previous studies have reported the averaged motility of hundreds of cells and not that of individual cells.

Here, we focused on studying the motility of individual diatom cells by using a two-dimensional (2D) dynamic image analysis software. Isolated cells of an adhesive diatom- Navicula sp.-were cultured on the surface of a glass slide in Petri dishes (diameter, $9 \mathrm{~cm}$ ) for 8 days at $18^{\circ} \mathrm{C}$ before observing their motility. Sterilized natural sea water $(35 \mathrm{ml}$ ) with Daigo IMK (Nihon Pharmaceutical Co. Ltd) was used as a culture medium. The living cells were directly observed using an inverse optical microscope under 5000 lux light at room temperature. $2 \mathrm{D}$ movements of the diatom cells for 5 to 10 minutes were captured as avi files in a computer, and velocity $\left(V_{x}, V_{y}\right)$, accerelation $\left(A_{x}\right.$ $A_{y}$ )angular momentum, and frequency of the movements were calculated for 13 individual cells in 3 independent experiments. We found that even in 1 Petri dish, the cells had 2 different velocity values. In addition, some of the cells showed a specific frequency in $V_{x}$ and $V_{y}$.

\section{P228 室温下での新生児ラット心筋細胞保存}

Preservation of neonatal rat cardiomyocytes at room temperature

Toshiki OKUDAIRA ${ }^{1}$, Masafumi NAGAYAMA ${ }^{1}$, Kazutoshi GOHARA ${ }^{1}$, Toshio TAIRA ${ }^{2}$, Kyoko SHIMIZU ${ }^{2}$, Masato SAKAI ${ }^{2}$, Tsutomu UCHIDA ${ }^{1},\left({ }^{1}\right.$ Division of Applied Physics, Graduate School of Engineering, Hokkaido University, Japan: ${ }^{2}$ Primary Cell Company Limited, Japan)

The demand of primary cultured cardiomyocytes increases recently for the development of the medicine for heart. Therefore, the preservation methods for cardiomyocytes are needed for their stable supply. Usually the isolated cardiomyocytes are delivered within 24 hours at about $4{ }^{\circ} \mathrm{C}$, but this condition is not enough to supply for long-distance transportation. In this study, we investigated the suitable temperature range for cardiomyocytes preservation for 72 hours with maintaining their normal functions.

Neonatal rat cardiomyocytes isolated in the culture medium were preserved at temperature under $0,3,10,20,23,30,35^{\circ} \mathrm{C}$. After 72 hours preservation, we observed the morphology of the preserved cardiomyocytes. We then cultured the preserved cells for 7 days to compare the extension process and beating properties of the preserved cells with those of non-preserved (control) cells. Cardiomyocytes preserved at temperatures higher than $30^{\circ} \mathrm{C}$ were closely aggregated during 72 hours storage. However, the cells preserved at temperatures below $20{ }^{\circ} \mathrm{C}$ maintained the initial isolated state. The microscopic observations indicated that these preserved cells extended normally, and beat regularly and spontaneously during culture. Furthermore, tendency of increase and decrease of beating rate during culture of preserved cells below $20^{\circ} \mathrm{C}$ was as similar as that of control. Therefore, we concluded that the cardiomyocytes can be preserved at temperature below $20{ }^{\circ} \mathrm{C}$ for 72 hours, which is more useful conditions than present commercial conditions.

1 229 細胞培養力学場設計に基づく遺伝子導入効率のメカニカル制御 Mechanical control of gene transfection efficiency on the welldefined elastic field of cell culture substrate

Hiraku Nakashima ${ }^{1}$, Tatsuya Okuda ${ }^{2}$, Thasaneeya Kuboki ${ }^{2}$, Satoru Kidoaki ${ }^{2},\left({ }^{1}\right.$ Department of Chemistry and Biochemistry, Graduate School of Engineering, Kyushu University: ${ }^{2}$ Institute for Materials Chemistry and Engineering, Kyushu University)

In general, cell functions are affected by the mechanical conditions of extracellular matrix or culture substrates. For example, substrate elasticity is known to regulate cell shape, proliferation, and differentiation. In addition, it has also been suggested that the conditions of mechanical field affect uptake efficiency of exogenous liquid factor. In particular, gene transfection efficiency has been reported to depend on the substrate elasticity [1], in which different elasticity substrates were prepared from different materials. To elucidate the mechanism relating to those kind of mechanical control of gene transfection efficiency, it is required to utilize the single-material-based gels with different elasticity, and fixed surface chemical conditions of matrix density and cell-adhesive molecule density for the systematic analysis of gene uptake and expression efficiency. For this issue, elasticity-tunable cell-adhesive gels with definite surface chemical conditions were prepared from photo-crosslinkable gelatin. 